\section{Micromanaging tolerance}

\section{By Kai-Jye Lou, Staff Writer}

Researchers at Northwestern University and the Myelin Repair Foundation have simplified an autologous cell-based strategy for promoting antigen-specific tolerance by replacing splenic leukocytes with synthetic microparticles as the antigen carrier. ${ }^{1}$ The groups are planning to first develop the therapy and establish clinical proof of concept in autoimmune indications for which the key antigen is known before moving forward in multiple sclerosis.

Apoptotic splenic leukocytes coupled to peptide antigens induce antigen-specific immune tolerance in T cell-mediated autoimmune diseases such as MS. ${ }^{2-4}$ Indeed, in a Phase I trial in patients with newonset relapsing-remitting MS (RRMS), researchers showed that infusion of such cells induced tolerance against the coupled myelin peptide antigens and did not cause significant adverse effects.

The trial was sponsored by the Myelin Repair Foundation (MRF) and the German government.

Despite the positive initial results, the MRF and Northwestern researchers wanted to simplify the approach and make it more amenable for commercialization as an off-the-shelf therapy. The cell therapy involves isolating patient leukocytes via leukapheresis, coupling the cells to peptide autoantigens via ethylene carbodiimide chemistry, rendering the cells apoptotic and then infusing the cells back into the patient.

"In the clinical trial, we found that we could infuse patients with up to three billion such cells without causing significant adverse effects and promote tolerance toward multiple myelin-associated antigens," said Stephen Miller, a professor and director of the Interdepartmental Immunobiology Center at Northwestern. "However, producing these cells is a very complex and expensive process."

Thus, the team sought to develop an alternative synthetic vehicle that could mimic the antigen-carrying role of the apoptotic splenic leukocytes and promote tolerance without the complexities associated with autologous cell therapies. The group settled on inert, negativelycharged carboxylated microparticles.

"We have shown that nanoparticles with certain surface properties can be anti-inflammatory but, more importantly, also home to the same place in the spleen as the dying, peptide-coupled cells," said Daniel Getts, a visiting research professor at Northwestern. "As such, we figured that we could use inert nanoparticles that the body potentially perceives as dying cells to deliver antigens and induce tolerance."

As proof of concept, the researchers used ethylene carbodiimide chemistry to attach an encephalitis-inducing peptide antigen to negatively charged poly(lactide-co-glycolide) (PLG) microparticles.

In a mouse model of experimental autoimmune encephalomyelitis (EAE), a single i.v. infusion of the peptide-linked microparticles induced tolerance to the attached peptide antigen and decreased symptom severity compared with infusion of microparticles linked to a control peptide.

The tolerogenic effects persisted for the duration of the mouse studies and depended on a scavenger receptor called macrophage receptor with collagenous structure (MARCO; SCARA2). The peptidelinked microparticles had no effect in mice lacking the receptor.

The peptide-linked microparticles work by homing to the splenic marginal zone, where they are taken up by MARCO-expressing macrophages. This in turn leads to a long-term $\mathrm{T}$ cell-mediated tolerogenic response specific to the peptide antigen.

Results were published in Nature Biotechnology. Getts is a colead author on the paper and Miller is a co-corresponding author.

"These inert microparticles could be formulated for use in a specific indication and then stored in a bottle on a shelf," said Jay Tung, chief research officer at MRF. "The microparticles are also easier to manufacture and characterize than a cell therapy and would thus have a more straightforward route for seeking FDA approval."

He added that the preclinical data have been independently validated by a CRO.

Miller said his group and the MRF are moving forward with the PLG microparticles. PLG is a biodegradable, FDAapproved polymer that is used in medical sutures and is amenable
"These inert microparticles could be formulated for use in a specific indication and then stored in a bottle on a shelf. The microparticles are also easier to manufacture and characterize than a cell therapy and would thus have a more straightforward route for seeking FDA approval." - Jay Tung, Myelin Repair Foundation to GMP manufacturing in large quantities. Moreover, PLG also is immunologically inert, so the particles themselves should not trigger or exacerbate an immune response.

"These peptide-linked microparticles appear to have a robust therapeutic effect in the preclinical models, and the tolerogenic response appears to be antigen specific, which is a good thing," said Howard Weiner, director of the Partners Multiple Sclerosis Center and co-director of the Center for Neurologic Diseases at Brigham and Women's Hospital. "None of the currently marketed drugs for multiple sclerosis works in an antigen-specific manner."

Weiner also said the persistent tolerogenic effect seen in the mouse models suggests the microparticles would not need to be dosed chronically.

Lawrence Steinman, professor of neurology and neurological sciences, pediatrics and genetics at the Beckman Center for Molecular and Genetic Medicine at the Stanford University School of Medicine, said immunomodulatory drugs for MS work by depleting T cells and/ 
or B cells and that chronic treatment with these immune cell-depleting therapies can have significant side effects, such as increased risk of infection.

He noted that antigen-specific strategies have the potential to trigger or exacerbate an autoimmune response and that future studies will need to monitor for such effects.

\section{Antigen ambiguity}

MRF and Northwestern now want to establish clinical proof of concept for the microparticle therapy in another autoimmune-related indication before attempting to develop it in MS.

Steinman said it all comes down to picking the relevant antigens.

"Diseases such as relapsing-remitting MS and secondary MS are going to be problematic because we still don't have a clear idea as to what the causal antigens are," he told SciBX. "Instead, I think that one should first try to develop these microparticles in autoimmune diseases where the causal antigens are known and no effective treatments are available. This is where the probability of success is going to be the highest."

He cited diseases such as neuromyelitis optica and myasthenia gravis. The former is caused by an autoimmune response against aquaporin 4 (AQP4) and the latter by an autoimmune response against nicotinic acetylcholine receptors (nAchRs).

Getts and Miller agreed that it may be easier to first develop the microparticles in an indication other than MS.

"What we don't want to have happen in clinical trials is to find out that the microparticles are ineffective because we didn't target the correct antigens," said Miller. "We want to first select a disease indication where we know the exact antigen to target."

Miller's group is also trying to show preclinical proof of principle in additional immune-mediated conditions, including type 1 diabetes, allergic airway disease and transplant rejection. He also said that the microparticles could be used as an adjunct to prevent an immune response against recombinant protein therapies for hemophilia.

Other possibilities, added Getts, include celiac disease and peanut allergy. "These are conditions where we have a reasonable handle on the precise antigens involved and can target them with great accuracy," he told SciBX.

Tung said MRF is in discussions to partner or out-license the technology for various indications. He said the foundation does plan to develop the microparticles for MS but acknowledged that it will be difficult to move forward in the indication until the manufacturing and analytical components of the therapy are better understood.

"MS is our primary mission, but it makes more sense to first demonstrate that the technology works in humans in a simpler system before tackling a problem as complex as MS," said Tung. "The beauty of this technology is that techniques that work out in simpler therapeutic areas will be applicable to MS."

MRF also plans to run additional studies to further characterize the microparticles and optimize their properties. He said the plan is to carry out such studies with an industry partner.

Tung said the microparticle technology is at least three years from an IND submission.

Northwestern and the MRF have pending patents covering the microparticles and their use for induction of antigen-specific tolerance. MRF has exclusive rights to license this technology in all therapeutic areas.

Lou, K.-J. SciBX 5(47); doi:10.1038/scibx.2012.1226

Published online Dec. 6, 2012

\section{REFERENCES}

1. Getts, D.R. et al. Nat. Biotechnol.; published online Nov. 18, 2012 doi:10.1038/nbt.2434

Contact: Stephen D. Miller, Northwestern University, Evanston, Illinois

e-mail: s-d-miller@northwestern.edu

2. Vanderlugt, C.L. et al. J. Immunol. 164, 670-678 (2000)

3. Turley, D.M. \& Miller, S.D. J. Immunol. 178, 2212-2220 (2007)

4. Getts, D.R. et al. J. Immunol. 187, 2405-2417 (2011)

COMPANIES AND INSTITUTIONS MENTIONED

Brigham and Women's Hospital, Boston, Mass.

Myelin Repair Foundation, Saratoga, Calif.

Northwestern University, Chicago, III.

Stanford University School of Medicine, Stanford, Calif. 